\title{
Joe Rosen: Lawless Universe
}

\author{
Fedde Benedictus
}

Received: 8 February 2011 / Accepted: 21 February 2011 / Published online: 3 March 2011

(C) The Author(s) 2011. This article is published with open access at Springerlink.com

"This book is a sequel to, a complement for, even an antidote for, books that create the misinterpretation that science can explain everything.” These bold words are used by Joe Rosen to preface his new book 'Lawless Universe'.

His central argument is that science is unable to describe the whole of reality. To support this statement, he starts by giving definitions of science, nature, reality and a variety of other concepts that are necessary for his exposition. But devising your own definitions is a bit like phrazing your own rules before playing the game-not too convincing. Rosen holds that scientific laws merely describe regularities in nature, and that our theories actually explain the occurrence of these regularities.

I am puzzled by this. In the case of a flying cannonball, the parabolic path it travels surely does not explain why it falls back down, but neither does the theory of gravity. Gravitas is simply the Latin word for weight! One may think that Einstein's curved spacetime now comes to the scientist's rescue. The fact that every mass simply follows a geodesic explains why objects appear to be heavy. But what is explained here? Why does every mass follow a geodesic? The argument should be obvious now. Einstein, too, did not explain gravity. His description of it was only more accurate than the descriptions available up till then.

Considering this line of argument more closely, the whole of science falls prey to its subversive influence. But Rosen does not consider this more closely. Instead of making the claim that science can never go beyond mere description, he presents us with the more modest claim that scientists are backing the wrong horse if they use the scientific method to describe the entire universe. He claims that parts of the universe can be described scientifically, but the whole of it is simply inaccessible to the

\footnotetext{
F. Benedictus ( $\varangle)$

Institute of History and Foundations of Science, Utrecht University, P.O. Box 80.010, 3508 TA

Utrecht, The Netherlands

e-mail: f.j.benedictus@uu.nl
} 
scientific method, as it is not reproducible-it is lawless. Rosen takes a particularly dim view of cosmology and the misguided search for a Theory Of Everything.

But we ought not panic. According to Rosen, the one phenomenon we can be perfectly sure of is our own existence. Therefore we may securely found our epistemology upon the anthropological principle. The problem with explanations of the anthropological kind is that most of us have the feeling that no explanation is being given, since there is no causation involved. This, Rosen holds, does not affect the principle's powers of implication. The nature of science forces us to look beyond science itself for explanations on a universal scale, and the subjective fundamentality of human existence makes this possible.

For me personally, Rosen's book provided an interesting read. The misinterpretation he describes in his preface is notorious, and claims like "If we do discover a theory of everything...it would be the ultimate triumph of human reason-for then we would truly know the mind of God." do indeed justify the administration of an antidote. Rosen's arguments provide us with a most welcome complement to books in which the scientific endeavour is elevated to dizzying heights.

Open Access This article is distributed under the terms of the Creative Commons Attribution Noncommercial License which permits any noncommercial use, distribution, and reproduction in any medium, provided the original author(s) and source are credited. 